\title{
No harm from angiotensin-converting enzyme inhibitors or angiotensin receptor inhibitors in patients with COVID-19. Results of a prospective study on a hospital-based cohort
}

\author{
Gian Paolo Anzola, ${ }^{1}$ Clara Bartolaminelli, ${ }^{2}$ Gina Alessandra Gregorini, ${ }^{3}$ Chiara Coazzoli, ${ }^{2}$ Francesca Gatti, ${ }^{4}$ \\ Alessandra Mora, ${ }^{4}$ Dimitrios Charalampakis, ${ }^{4}$ Andrea Palmigiano, ${ }^{4}$ Michele De Simone, ${ }^{4}$ Alice Comini,${ }^{4}$ \\ Erica Dellaglio, ${ }^{4}$ Salvatore Cassetti, ${ }^{4}$ Maurizio Chiesa, ${ }^{4}$ Francesca Spedini, ${ }^{4}$ Patrizia d'Ottavi, ${ }^{2}$ Maria Cristina Savio ${ }^{4}$ \\ ${ }^{1}$ San Camillo Hospital, Brescia; ${ }^{2}$ Azienda Territoriale Sanitaria (Territorial Health Authority), Brescia; ${ }^{3}$ University of Brescia; \\ ${ }^{4}$ Emergency Department, Gavardo Hospital, Brescia, Italy
}

\begin{abstract}
This study aims to assess the effect of angiotensin-converting enzyme inhibitors (ACEIs) and angiotensin receptor inhibitors (ARBs) on the course of COVID-19. It is a prospective study on $221(\mathrm{M} / \mathrm{F}$ ratio=143/78, mean age 72 \pm 13 ) consecutive hypertensive patients with COVID-19: 76 (34.4\%) treated with ACEIs, 63 (28.5\%) with ARBs and 82 (37.1\%) with antihypertensives OTHER than ACEIs or ARBs. They were all followed up until discharge or death. BAD outcome was defined as the need for invasive mechanical ventilation or death. The three classes of medication were well balanced for confounding variables. BAD outcome was overall recorded in 63/221 (28\%) patients, in $20 / 76(26 \%)$ of ACEI, in $17 / 63$ (27\%) of ARB and in 26/82 $(32 \%)$ of OTHER users, with no statistically significant dif-

Correspondence: Gian Paolo Anzola, Consultant Neurologist, San Camillo Hospital, via Turati 47, 25123 Brescia, Italy.

Tel.: +39.030.2910311. E-mail: gpanzola@gmail.com ference in any comparison. These findings refute the hypothesis that treatment with ACEIs or ARBs may negatively affect the course of COVID-19.
\end{abstract}

Key words: COVID-19; angiotensin-converting enzyme inhibitors; angiotensin receptor inhibitors.

Acknowledgments: Gian Paolo Anzola, Clara Bartolaminelli, Gina Alessandra Gregorini, and Chiara Coazzoli wish to publicly manifest their admiration and gratitude towards all the health professionals in the Emergency Department of the Gavardo Hospital who struggle daily against COVID-19 to look after patients at the risk of their own lives.

Funding: the present study was undertaken with no external funding and was conducted voluntarily by all participants for humanitarian reasons.

Contributions: GPA made the statistical analysis and wrote the first draft of the paper; $\mathrm{CB}$ conceived the study and helped to collect data; GAG gave conceptual support; CC, FG, AM, DC, AP, MDS, AC, ED, SC, MC, FS, PdO and MCS collected data.

Conflict of interests: the authors declare no potential conflict of interests.

Availability of data and materials: original data stored in the electronic files of the Gavardo Hospital.

Ethics approval and consent to participate: the study was approved by the Local Institutional review Board of the Gavardo Hospital.

Received for publication: 10 May 2020.

Revision received: 8 June 2020.

Accepted for publication: 8 June 2020.

This work is licensed under a Creative Commons Attribution NonCommercial 4.0 License (CC BY-NC 4.0).

${ }^{\circ}$ Copyright: the Author(s), 2020

Licensee PAGEPress, Italy

Italian Journal of Medicine 2020; 14:162-166

doi:10.4081/itjm.2020.1313

\section{Introduction}

Following the outbreak of coronavirus disease 19 (COVID-19) considerable concern has emerged for the potential harm in the use of renin-angiotensin system (RAS) inhibitors, namely angiotensin-converting enzyme inhibitors (ACEIs) and angiotensin receptor inhibitors (ARBs), given that ACEIs and ARBs may increase the expression of ACE2 receptors that represent the way for coronavirus 2 (SARS$\mathrm{CoV}-2$ ) to enter into the cell and cause severe acute respiratory syndrome. ${ }^{1}$

Partly to help to solve this issue we undertook a prospective study aimed at assessing the clinical characteristics, with particular emphasis on the type of antihypertensive medication, of all consecutive patients presenting at the Emergency Department of a Community Hospital in Gavardo, in the neighborhood of Brescia in Lombardy (Italy), and found to be positive for SARS-CoV-2 infection. We report here the findings on hypertensive patients. The whole cohort will be the subject of a further paper.

\section{Materials and Methods}

From March 12 to April 12, 2020, all consecutive patients presenting at the Emergency Department of 
the Community Hospital of Gavardo for symptoms or signs suggestive of SARS-CoV-2 infection were considered for the study. However, only those confirmed by a real-time polymerase chain reaction in nasal or pharyngeal swab were included.

Following a pre-specified protocol, relevant clinical and laboratory variables were recorded on the field, focusing on the type of current anti-hypertensive treatment. In particular, a dichotomous categorization (yes/no) was employed for cardiovascular disease (CVD) (including the history of myocardial infarction, percutaneous transcatheter coronary angioplasty, coronary artery by-pass grafting, heart failure stroke and atrial fibrillation), chronic renal failure, chronic liver disease, diabetes mellitus, chronic obstructive pulmonary disease, history of or present neoplasm, history of or present autoimmune disease, hypertension, current use of ACEIs, current use of ARBs, current use of antihypertensives other than ACEIs or ARBs (OTHERs). Relevant laboratory tests were recorded at presentation: these included hemoglobin in $\mathrm{g} / \mathrm{L}$, platelet, leukocyte and lymphocyte count per microliter, serum creatinine in $\mathrm{mg} / \mathrm{dL}$, aspartate aminotransferase (AST), alanine aminotransferase in units per liter and C-reactive protein $(\mathrm{CRP})$ in $\mathrm{mg} / \mathrm{L}$. In case of missing data, the electronic chart was reviewed on discharge to finalize ascertainment.

Endpoints were: GOOD outcome if during the hospital stay, the patient survived and did not need invasive mechanical ventilation and BAD outcome if he/she needed invasive mechanical ventilation or died. In non-hospitalized patients, a follow-up telephone call was done three weeks after the presentation.

Single comparisons were performed with Chisquare test or Fisher's exact test when appropriate on categorical variables and with independent sample T-test (if normally distributed) or with the MannWhitney U test on continuous variables. Variables that had proven significant on univariate analysis were included to multivariate binomial logistic regression analyses to identify independent predictors of BAD outcome. Statistical significance was set at $\mathrm{P}<0.05$. SPSS 20 statistical package was used.
The Local Institutional Review Board approved the study.

\section{Results}

From the entire cohort of 431 patients who accessed the Emergency Department during the study period, $221(51 \%, \mathrm{M} / \mathrm{F}$ ratio $=143 / 78$, mean age $72 \pm 13$ ) were on ongoing treatment for hypertension, 76 (34.4\%) with ACEIs, 63 (28.5\%) with ARBs and $82(37.1 \%)$ with antihypertensives OTHER than ACEIs or ARBs. Overall, 171/221 (78\%) subjects were hospitalized. The outcome was GOOD in 158/221 (72\%) patients and BAD in 63/221 (28\%).

Compared with patients with GOOD outcome, those with $\mathrm{BAD}$ outcome were significantly older (79 \pm 9 vs $69 \pm 13, \mathrm{P}<0.0001$ ), had more severe markers of inflammation, as shown by CRP ( $160 \pm 82$ vs $89 \pm 70$, $\mathrm{P}<0.0001)$ and of liver and kidney failure as expressed by AST $(64 \pm 50$ vs $46 \pm 24, \mathrm{P}<0.0001)$ and creatinine levels $(1.78 \pm 1.3$ vs $1.08 \pm 0.4, \mathrm{P}<0.0001)$ respectively. There was a trend for female patients to fare better than males $(20 / 78,26 \%$ bad outcome in females, $v s$ $43 / 143,30 \%$ bad outcome in males), but the difference was not statistically significant. On univariate analysis of dichotomized variables, BAD outcome was statistically associated with age $\geq 70$ years, presence of CVD, creatinine $\geq 1 \mathrm{mg} / \mathrm{dL}, \mathrm{AST} \geq 32 \mathrm{U} / \mathrm{L}$ and lymphocyte count $\leq 1000 / \mathrm{mL}$ (Table 1 ).

Variables that turned significant in univariate analyses were included into a multivariate binomial logistic regression analysis with $\mathrm{BAD}$ outcome as the dependent variable. Only age, CRP, and creatinine remained independent predictors of BAD outcome (Table 2). With regard to age, BAD outcome was recorded in $4 \%$ of subjects younger than $60,18 \%$ of those in the $61-70$ range, $35 \%$ of those between 71 and 80 , and in $48 \%$ of those older than 80 .

The case fatality rate was overall at $23 \%(51 / 221)$. Among ACEI users, it was 20\% (15/61), among ARB $22 \%(14 / 49)$ and among OTHER users 27\% (22/60) with no statistically significant difference in any comparison (Table 3).

Table 1. The impact of significant variables on the outcome, expressed as relative risk, in univariate analysis.

\begin{tabular}{lccc}
\hline & RR for BAD outcome & $\mathbf{9 5 \%}$ CI & P \\
\hline Age $\geq 70$ & 3.59 & $1.93-6.66$ & $<0.0001$ \\
\hline CVD $($ composite) & 2.24 & $1.50-3.30$ & $<0.0005$ \\
\hline Creatinine $\geq 1$ & 2.54 & $1.58--4.07$ & $<0.0001$ \\
\hline Lymphocyte $\leq 1100 / \mathrm{mL}$ & 1.71 & $1.01-2.90$ & $<0.05$ \\
\hline AST $\geq 32 \mathrm{U} / \mathrm{L}$ & 2.63 & $1.33-5.21$ & $<0.005$ \\
\hline
\end{tabular}

RR, relative risk; CI, confidence interval; CVD, cardiovascular disease; AST, aspartate aminotransferase 
Table 2. Logistic regression analysis with BAD outcome as a dependent variable.

\begin{tabular}{|c|c|c|c|c|c|c|c|c|}
\hline & \multirow[t]{2}{*}{$\beta$} & \multirow[t]{2}{*}{ SE } & \multirow[t]{2}{*}{ Wald } & \multirow[t]{2}{*}{ df } & \multirow[t]{2}{*}{ Sig. } & \multirow[t]{2}{*}{ OR } & \multicolumn{2}{|c|}{ 95\% CI for OR } \\
\hline & & & & & & & Lower & Upper \\
\hline Age & 0.076 & 0.020 & 13.932 & 1 & 0.000 & 1.079 & 1.037 & 1.123 \\
\hline AST U/L & 0.011 & 0.007 & 2.519 & 1 & 0.112 & 1.011 & 0.997 & 1.024 \\
\hline $\mathrm{CRP} \mathrm{mg} / \mathrm{L}$ & 0.011 & 0.003 & 12.976 & 1 & 0.000 & 1.011 & 1.005 & 1.016 \\
\hline Creatinine $\mathrm{mg} / \mathrm{dL}$ & 0.920 & 0.308 & 8.936 & 1 & 0.003 & 2.510 & 1.373 & 4.589 \\
\hline Lymphocyte count $\leq 1000 / \mathrm{mm}$ & 0.000 & 0.000 & 0.239 & 1 & 0.625 & 1.000 & 0.999 & 1.001 \\
\hline CVD & 0.624 & 0.417 & 2.237 & 1 & 0.135 & 1.866 & 0.824 & 4.225 \\
\hline RAS inhibitors (ACEI or ARB) & 0.460 & 0.421 & 1.190 & 1 & 0.275 & 1.584 & 0.693 & 3.618 \\
\hline Costante & -9.989 & 1.840 & 29.484 & 1 & 0.000 & 0.000 & - & - \\
\hline
\end{tabular}

$\beta$, beta coefficient; SE, standard error; df, degree of freedom; Sig., significance; OR, odds ratio; CI, confidence interval; AST, aspartate aminotransferase; CRP, C-reactive protein; CVD, cardiovascular disease; RAS, renin-angiotensin system; ACEI, angiotensin-converting enzyme inhibitor; ARB, angiotensin receptor inhibitor.

Table 3. Demographic, clinical and outcome findings in patients treated with ACEI, ARB and OTHER antihypertensive drugs.

\begin{tabular}{|c|c|c|c|c|c|}
\hline & $\begin{array}{c}\text { All } \\
221(100)\end{array}$ & $\begin{array}{c}\text { ACEI } \\
76(34.4)\end{array}$ & $\begin{array}{c}\text { ARB } \\
63(28.5)\end{array}$ & $\begin{array}{c}\text { Other } \\
82(37.1)\end{array}$ & $\mathbf{P}$ \\
\hline Age & $72 \pm 13$ & $70 \pm 14$ & $70 \pm 12$ & $74 \pm 12$ & n.s. \\
\hline Male/Female & $143 / 78$ & $54 / 22$ & $34 / 29$ & $55 / 27$ & n.s. \\
\hline CVD & $61 / 221(28)$ & $23 / 76(30)$ & $16 / 63(25)$ & $22 / 82(27)$ & n.s. \\
\hline Creatinine $\geq 1$ & $102 / 221(47)$ & $35 / 76(46)$ & $30 / 63(48)$ & $37 / 82(45)$ & n.s. \\
\hline Creatinine $\mathrm{mg} / \mathrm{dL}$ & $1.27 \pm 0.8$ & $1.18 \pm 0.5$ & $1.28 \pm 0.7$ & $1.36 \pm 1.15$ & n.s. \\
\hline CRP & $109 \pm 80$ & $99 \pm 55$ & $104 \pm 83$ & $122 \pm 95$ & n.s. \\
\hline AST & $51 \pm 34$ & $45 \pm 22$ & $54 \pm 49$ & $53 \pm 30$ & n.s. \\
\hline Lymphocyte count & $985 \pm 476$ & $1038 \pm 372$ & $1010 \pm 455$ & $918 \pm 564$ & n.s. \\
\hline GOOD outcome & $158(72)$ & $56(74)$ & $46(73)$ & $56(68)$ & n.s. \\
\hline BAD outcome & $63(28)$ & $20(26)$ & $17(27)$ & $26(32)$ & n.s. \\
\hline
\end{tabular}

Data show mean (standard deviation) for continuous or n. (\%) for categorical variables. ACEI, angiotensin-converting enzyme inhibitor; ARB, angiotensin receptor inhibitor; Other, antihypertensive other than ACEI or ARB; n.s., not significant; CVD, cardiovascular disease (any among ischemic heart disease, heart failure, atrial fibrillation, stroke); CRP, Creactive protein; AST, aspartate aminotransferase; GOOD outcome, alive at the end of hospitalization and with no need of invasive mechanical ventilation; BAD outcome, dead or in need of invasive mechanical ventilation.

Variables that on univariate analyses were significant in predicting a BAD outcome were evenly distributed across classes, as shown in Table 3. Likewise, age and gender were well balanced. Bad outcome was recorded in $26 \%$ of ACEI, $27 \%$ of ARB, and $32 \%$ of OTHER users, again with no statistically significant difference.

\section{Discussion and Conclusions}

The results of the present study show that in COVID-19 hypertensive patients presenting at the Emergency Unit the outlook is somewhat unfavorable, as the proportion of individuals who will progress to invasive mechanical ventilation or will eventually die is $28 \%$ on the average but with a steep rise to $35 \%$ in people older than 70 and to $48 \%$ in older than 80 . This finding is in agreement with previous reports that have identified hypertension and age as main predictors of bad outcome or death in hospitalized patients. ${ }^{2-4}$

Cardiovascular disease, which in univariate analysis appeared to double the risk of BAD outcome, was no longer significant when corrected by age, again in agreement with previously published findings, ${ }^{4}$ whereas, creatinine and CRP remained independent predictors of bad outcome, most likely as markers of the systemic involvement of infection.

With regard to the main aim of the study, ACEIs and ARBs each accounted for $30 \%$ of the cohort of 
hypertensive patients, the remaining drugs being represented for the vast majority by diuretics, betablockers, and calcium antagonists. These were put together under the heading of OTHER, for comparison with ACEIs and ARBs. From Table 3 it appears that most if not all confounders were equally balanced among classes, and the bad outcome was equally frequent in ACEI, ARB, and OTHER. The same holds for the case fatality rate. Concerning patients with a BAD outcome, there was a trend for an over-representation of OTHER $(26 / 63,41 \%)$ with respect to ACEI $(20 / 63,32 \%)$ and ARB $(17 / 63,27 \%)$ but the trend was not statistically significant. Finally, ACEIs/ARBs put together were excluded by logistic regression analysis as predictors of bad outcome.

All together these findings refute the hypothesis that treatment with ACEIs or ARBs may negatively affect the course of COVID-19, in agreement with the results of three retrospective studies done in hospitalized patients in China. ${ }^{5-7}$ However, essential differences with regard to these studies need to be noted: age was lower in one, ${ }^{5}$ the proportion of ACEI/ARB users was much lower than ours in another ${ }^{6}$ and, the most important thing, these studies were performed only on Asian patients, who are usually treated with lower doses of antihypertensive medication and are known to metabolize $\beta$-blockers differently from Caucasians. ${ }^{8}$ Therefore, the results of these studies may not be entirely transferable to western patients. Nevertheless, our findings are quite similar and show substantial neutrality of ACEIs and ARBs on the outcome. We noted a non-significant trend for a worse outcome in users of OTHER antihypertensives, which compares with Zhang's finding of a protective effect of ACEI. ${ }^{6}$ However, it is important to note that Zhang et al. compared the in-hospital use of ACA/ARBs with other medications and were unable to assess pre-hospital treatment. ${ }^{6}$ This may, of course, have introduced a critical bias. Yet, a protective effect of ACEIs on death is suggested from the findings of a recently published study performed in 8910 hospitalized patients. ${ }^{9}$ This study included subjects of different ethnicities from all over the world, and the proportion of patients taking ACEIs or ARBs was extremely low. Therefore, according to what the Authors themselves admitted, this conclusion must be taken very cautiously. ${ }^{9}$

On the other hand, further support for the substantial safety of RAS inhibitors comes from two population-based studies that were unable to find any association between ACEI or ARB use and the susceptibility to COVID-19 or the likelihood of a worse outcome in affected subjects. ${ }^{10,11}$

In summary, regardless of the study type, sample size, and outcome variables, the bulk of studies that have appeared in the last few weeks unanimously re- ject the hypothesis that taking ACEIs or ARBs adversely affects the course of COVID-19. This conclusion is confirmed by the results of the present study, which is the only one, to the authors' knowledge, to have assessed patients prospectively on the field from Emergency Unit to discharge or death.

The whole picture emerging from available data plays in favor of the safety of RAS inhibitors, with a hint for a possible protective effect from ACEIs, which, however, will have to be tested in future randomized controlled trials. For the time being, a conservative conclusion may suggest that there is no reason to withhold ACEIs and ARBs in patients with COVID-19.

Our study suffers from several limitations: it was based on a single-center experience, and the duration of treatment or the dosage of drugs could not be assessed. Furthermore, we are aware that the relatively small sample size might expose to a Type II error in erroneously accepting the null hypothesis. We did not perform a calculation of the required sample size, but with a prevalence of the outcome variable of $28 \%$, the sample size needed to pick up a difference of $12 \%$ in its occurrence would have been 486 . Finally, the three classes of medication were well balanced for confounding variables, and we believe that any minimal difference that could emerge from a more powerful study would probably be clinically meaningless.

\section{References}

1. Danser AHJ, Epstein M, Batlle D. Renin-angiotensin system blockers and the COVID-19 pandemic: at present there is no evidence to abandon renin-angiotensin system blockers. Hypertension 2020 [Epub ahead of print].

2. Guan WJ, Ni ZY, Hu Y, et al. Clinical characteristics of coronavirus disease 2019 in China. N Engl J Med 2020 [Epub ahead of print] .

3. Wu C, Chen X, Cai Y, et al. Risk factors associated with acute respiratory distress syndrome and death in patients with coronavirus disease 2019 pneumonia in Wuhan, China. JAMA Intern Med 2020. [Epub ahead of print]

4. Zhou F, Yu T, Du R, et al. Clinical course and risk factors for mortality of adult inpatients with COVID-19 in Wuhan, China: a retrospective cohort study. Lancet 2020;395:1054-62.

5. Li J, Wang X, Chen J, et al. Association of renin-angiotensin system inhibitors with severity or risk of death in patients with hypertension hospitalized for coronavirus disease 2019 (COVID-19) Infection in Wuhan, China. JAMA Cardiol 2020. [Epub ahead of print]

6. Zhang P, Zhu L, Cai J, et al. Association of inpatient use of angiotensin converting enzyme inhibitors and angiotensin II receptor blockers with mortality among patients with hypertension hospitalized with COVID-19. Circ Res 2020. [Epub ahead of print] 
7. Meng J, Xiao G, Zhang J, et al. Renin-angiotensin system inhibitors improve the clinical outcomes of COVID19 patients with hypertension. Emerg Microbes Infect 2020. [Epub ahead of print]

8. Connelly Kudzma E. Cultural competence: cardiovascular medications. Prog Cardiovasc Nurs 2001; 16:4.

9. Mehra MR, Desai SS, Kuy SR, et al. Cardiovascular dis- ease, drug therapy, and mortality in Covid-19. NEJM 2020. [Epub ahead of print]

10. Reynolds HR, Adhikari S, Pulgarin C, et al. Renin-angiotensin-aldosterone system inhibitors and risk of Covid-19. NEJM 2020. [Epub ahead of print]

11. Mancia G, Rea F, Ludergnani M, et al. Renin-angiotensin-aldosterone system blockers and the risk of Covid-19. NEJM 2020. [Epub ahead of print] 